\title{
Modulation of the ionosphere by Pc5 waves observed simultaneously by GPS/TEC and EISCAT
}

\author{
V. Belakhovsky ${ }^{1,4}$, V. Pilipenko $2,4^{*}$, D. Murr ${ }^{3}$, E. Fedorov ${ }^{4}$ and A. Kozlovsky ${ }^{5}$
}

\begin{abstract}
Earlier studies demonstrated that the monitoring of the ionospheric total electron content (TEC) by global satellite navigation systems is a powerful method to study the propagation of transient disturbances in the ionosphere, induced by internal gravity waves. This technique has turned out to be sensitive enough to detect ionospheric signatures of magnetohydrodynamic waves as well. However, the effect of TEC modulation by ULF waves is not well examined as a responsible mechanism has not been firmly identified. During periods with intense Pc5 waves distinct pulsations with the same periodicity were found in the TEC data from high-latitude GPS receivers in Scandinavia. We analyze jointly responses in TEC variations and EISCAT ionospheric parameters to global PC5 pulsations during the recovery phase of the strong magnetic storms on October 31, 2003. Comparison of periodic fluctuations of the electron density at different altitudes from EISCAT data shows that main contribution into TEC pulsations is provided by the lower ionosphere, up to $\sim 150 \mathrm{~km}$, that is the E-layer and lower F-layer. This observational fact favors the TEC modulation mechanism by field-aligned plasma transport induced by Alfven wave. Analytical estimates and numerical modeling support the effectiveness of this mechanism. Though the proposed hypothesis is basically consistent with the analyzed event, the correspondence between magnetic and ionospheric oscillations is not always perfect, so further studies need to be conducted to understand fully the TEC modulations associated with Pc5 pulsations.
\end{abstract}

Keywords: Ionosphere, GPS, TEC, EISCAT, ULF waves, PC5 pulsations, Alfven waves

\section{Introduction}

The ionosphere represents an inner boundary of the nearEarth environment where the energy exchange occurs between the neutral atmosphere and the plasma of outer space. MHD waves provide an effective channel of the energy transfer from the outer magnetosphere to the bottom of the ionosphere. The interaction between the solar wind and magnetosphere acts as a permanent source of various types of MHD waves in the ultra-low-frequency (ULF) band, which fill the entire magnetosphere and reach its inner boundary, the ionosphere. While ground magnetometers and magnetospheric satellites provided tremendous amount of information about ULF wave properties in the magnetosphere and on the ground, the

\footnotetext{
*Correspondence: space.soliton@gmail.com

2 Space Research Institute, Moscow, Russia

Full list of author information is available at the end of the article
}

wave properties in the ionosphere remained unavailable to in situ observations. Low-Earth orbit satellites can detect a high-frequency part only of ULF spectrum (Pc1-3 waves). Ionospheric signatures of long-period ULF waves (Pc4-5, Pi2-3) can be detected by modern HF radio sounding techniques: Doppler sounders (Menk et al. 2007; Waters et al. 2007; Pilipenko et al. 2013) and SuperDARN HF radars (Lester et al. 2000; Ponomarenko et al. 2001; Teramoto et al. 2014). The ever-growing array of global satellite navigation systems (GPS, GLONASS, etc.) provide information on variations of a radiopathintegrated ionospheric parameter-the total electron content (TEC). GPS/TEC observations are becoming a powerful tool to monitor the propagation of acoustic and internal gravity waves along the ionosphere (Afraimovich et al. 2013; Komjathy et al. 2012).

The GPS/TEC technique turned out to be sensitive enough to detect ionospheric signatures of ULF waves 
as well. Early results, during the era of Faraday technique with geostationary beacons, reported TEC fluctuations related to geomagnetic variations in the Pc3-4 range (30to 50-s period) (Davies and Hartman 1976; Okuzawa and Davies 1981). The TEC modulation by intense Pc5 pulsations was found by Pilipenko et al. (2014a) and Watson et al. (2015). Thus, the standard TEC/GPS technique is sufficiently sensitive to detect ULF waves in some cases.

However, a physical mechanism of TEC periodic modulation associated with ULF waves has not been established yet. Additional periodic ionization and consequently TEC variations may occur owing to the ULFmodulated precipitation of energetic electrons into the ionosphere (Watson et al. 2015). Other possible mechanisms of TEC modulation by incident MHD waves comprise (a) plasma compression by evanescent fast compressional mode wave arising from the interaction of an Alfven wave with the anisotropic ionosphere (Pilipenko and Fedorov 1995); (b) periodic advection across a lateral gradient of the ionospheric plasma (Waters and Cox 2009); (c) periodic vertical shift and reconfiguration of the plasma vertical profile (Poole and Sutcliffe 1987); (d) frictional heating of ionospheric ions owing to periodic dragging through neutrals, and (e) field-aligned plasma transport by an Alfven wave (Pilipenko et al. 2014a). These possible mechanisms of TEC modulation by magnetospheric ULF waves provide main contribution either to the upper ionosphere (F-layer) or to the lower ionosphere (E-layer and bottom of F-layer). A possibility to reveal a contribution of ionospheric plasma density oscillations at different altitudes into the total TEC fluctuations may help to identify a responsible mechanism.

Here we analyze a unique event when the same global Pc5 waves were detected in the ionosphere by the GPS/ TEC technique (Pilipenko et al. 2014a) and EISCAT radar (Pilipenko et al. 2014b). We analyze these observations simultaneously which has provided an additional information on the relationship between geomagnetic and ionospheric variations. Observational results are validated by numerical modeling of Alfven wave interaction with a realistic ionosphere profile.

\section{Observational data}

We use the standard TEC data with 30-s resolution from an array of GPS receivers in Scandinavia (see map in Fig. 1). Dual-frequency GPS method uses the phase information from radio signals transmitted from GPS satellites at the L1 (1575.42 MHz) and L2 (1227.60 MHz) frequencies for estimating the slant TEC. The slant TEC along a radiopath can be converted into the vertical vTEC, denoted here as $N_{\mathrm{T}}$, by assuming the altitude of pierce points to be $250 \mathrm{~km}$. As a measure of columnar density $N_{\mathrm{T}}$ the TEC unit $\left(1 \mathrm{TECu}=10^{16} \mathrm{~m}^{-2}\right)$ is used.
Magnetometer 10-s data from the IMAGE array, covering the range of geographic latitudes from $\sim 79^{\circ}$ to $\sim 58^{\circ}$, are used (Fig. 1). The magnetometer observations are augmented with the multi-beam IRIS riometer data from Kilpisjarvi (KIL) that monitor a cosmic noise absorption caused by the energetic $(>30 \mathrm{keV})$ electron precipitation into the ionosphere. The magnetometer data have been decimated to a common 30-s step with TEC data.

We use the data with 30-s cadence from the UHF radar EISCAT, comprising the receivers at Sodankyla (SOD) and Kiruna (KIR), and receiver-transmitter at Tromso (TRO) (Fig. 1). EISCAT radar beam was directed along the geomagnetic field line. Intersection of receiving paths from SOD and KIR is located nearly above the magnetic station TRO (geographic coordinates $68.0^{\circ} \mathrm{N}, 19.1^{\circ} \mathrm{E}$ ) at altitude $\sim 290 \mathrm{~km}$. This radar system enables one to determine the vector of the ionospheric plasma drift velocity $\mathbf{V}$ and corresponding electric field E. The EISCAT radar system also measures the altitude profile of electron density $N_{\mathrm{e}}(z)$, ion temperature $T_{\mathrm{i}}(z)$, and electron temperature $T_{\mathrm{e}}(z)$ along the beam up to $\sim 400 \mathrm{~km}$.

\section{October 31, 2003, ULF event}

During the recovery phase of large magnetic storm on October 31, 2003, very intense (up to a few hundred nT) global quasi-monochromatic Pc5 waves were observed (Kleimenova and Kozyreva 2005). Typically, global Pc5 waves are observed during high solar wind streams, and they are about an order of magnitude more intense than common Pc5 pulsations. The reason for such outstanding intensity has not been found yet. Global Pc5 waves are coherent over a wide range of geomagnetic latitudes, during morning and post-noon hours (Potapov et al. 2006). Detailed studies of this event indicated that global Pc5 pulsations are probably caused by oscillations of the magnetospheric MHD waveguide, engulfing an entire magnetosphere, up to equatorial latitudes (Marin et al. 2014). At high latitudes, magnetospheric waveguide oscillations are strongly coupled with field line Alfven oscillations (Pilipenko et al. 2012).

During the periods with elevated Pc5 activity, 11001200 and 1200-1300 UT, TEC fluctuations have been compared with ground geomagnetic variations at station KIR (geographic latitude $67.8^{\circ}$ ) and ionospheric parameters determined by EISCAT radar. The tracks of intersection with the ionosphere of radio paths from satellites GPS7 and GPS9 to ground GPS receivers KIRU, VARS, and TROM (pierce points) are shown in Fig. 1.

The TEC data show gradual variations around 30-40 TECu with superposed small-scale fluctuations. To highlight these fluctuations the TEC data have been detrended with a cutoff frequency of $1 \mathrm{mHz}$. 


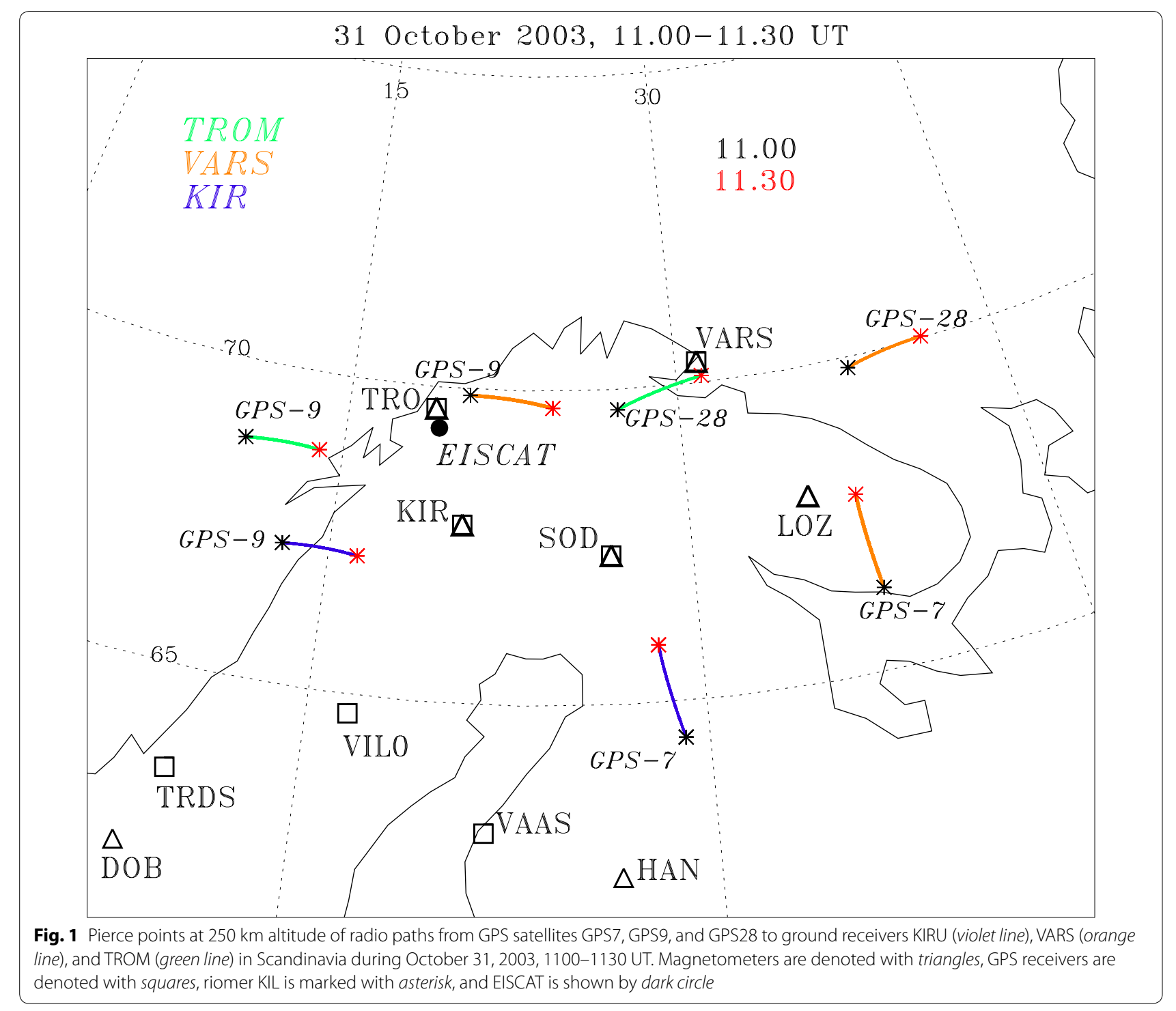

Quasi-periodic TEC pulsations have been revealed over a wide latitudinal range. The comparison for the period 1100-1330 UT of TEC fluctuations along paths GPS7/ KIRU, GPS9/KIRU with magnetic variations at KIR and EISCAT-derived ionospheric density $N_{\mathrm{e}}$ in the lower ionosphere, shows the occurrence of persistent periodicity in all these parameters (Fig. 2). The peak-to-peak amplitudes of oscillations of the TEC are $\Delta N_{\mathrm{T}} \sim 0.6$ TECU (GPS7/KIRU), and $1.0 \mathrm{TECU}$ (GPS9/KIRU), and magnetic pulsations $\Delta B \sim 400 \mathrm{nT}$ ( $X$ component) at KIR. According to visual inspection the phase relation between magnetic ( $X$ component) and TEC variations is not very stable: It varies from roughly out-of-phase during $\sim 1100-1200$ and $\sim 1215-1235$ UT to roughly inphase during 1200-1215 and 1305-1330 UT.
At the same time, the riometer data do not demonstrate the periodicity evident in magnetometer data (bottom panel in Fig. 2). Just few peak-to-peak correspondence may be found, representing weak signatures of ULF modulation of energetic electron precipitation.

Spectral analysis confirmed the occurrence of the same periodicity with $f \sim 2.4 \mathrm{mHz}$ in variations of the geomagnetic field, TEC (GPS7, GPS9), and EISCAT $N_{\mathrm{e}}$ (Pilipenko et al. 2014a, b). Cross-spectral analysis also showed a good correspondence between TEC and $B$ variations. During the 1130-1300 UT time interval the spectral coherency of TEC fluctuations at GPS9/KIRU and magnetic pulsations at KIR around the frequency $2.5 \mathrm{mHz}$ was high, $\gamma(f) \sim 0.8$. The ratio between the spectral densities of TEC and $X$-component magnetic variations at 
31 October 2003

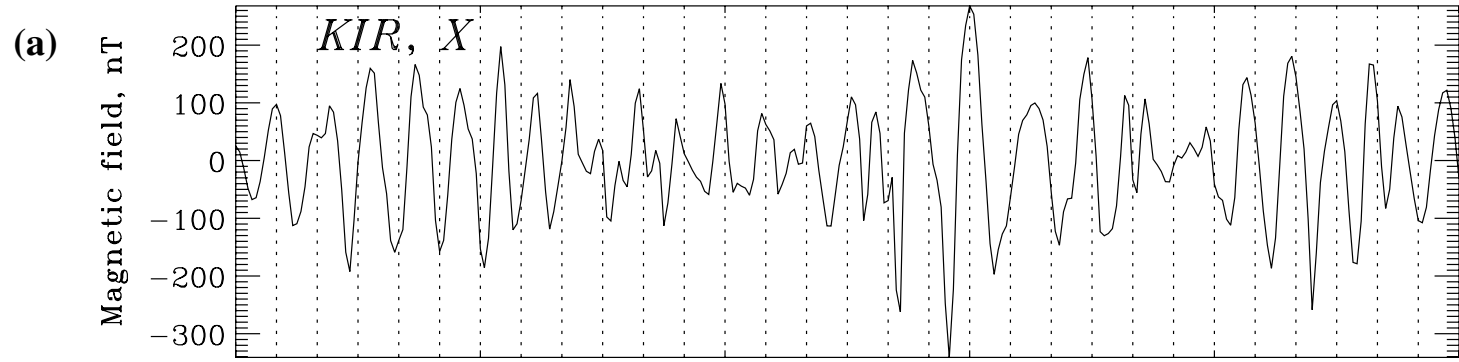

(b)

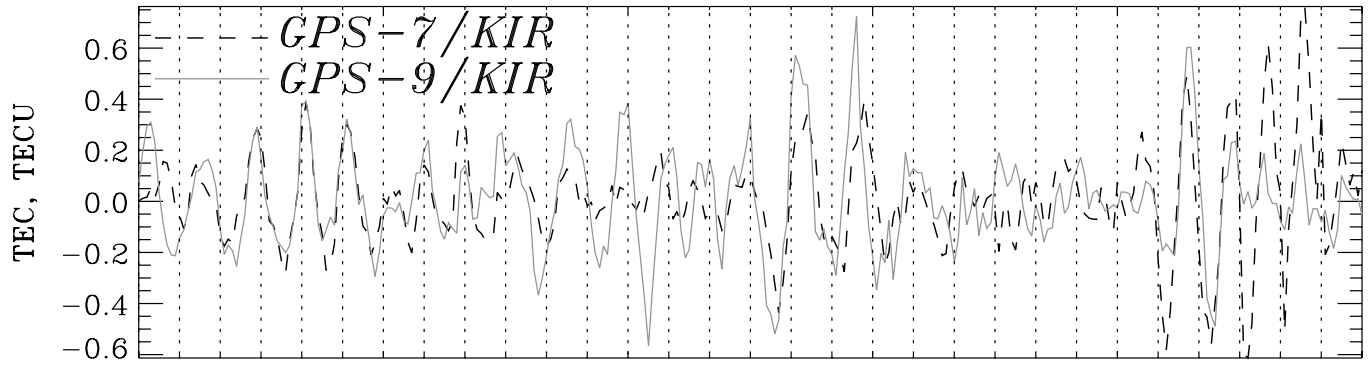

(c) $2.5 \times 10^{11}=E I S C A T$
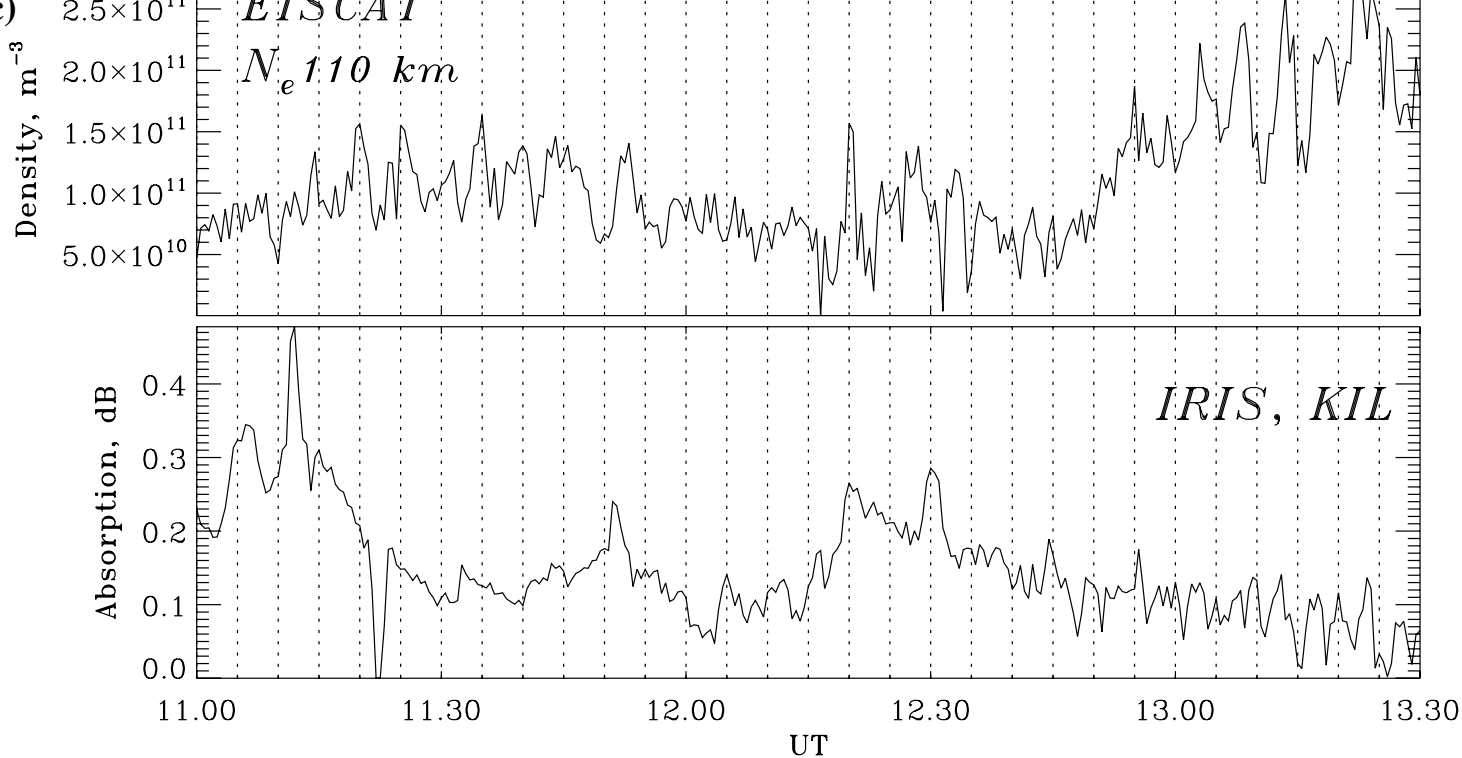

Fig. 2 Multi-instrument observations of Pc5 waves during October 31, 1100-1330 UT: a X component (in nT) of geomagnetic pulsations at KIR, b detrended (with a 1-mHz cutoff frequency) TEC fluctuations (in TECu) along radio paths GPS7/KIRU (dotted line) and GPS9/KIRU (solid line), c EISCAT $N_{\mathrm{e}}$ fluctuations at $h=110 \mathrm{~km}$; and $\mathbf{d}$ cosmic noise absorption from KIL riometer

this frequency was $\Delta N_{\mathrm{T}}(f) / \Delta B(f) \sim 2 \times 10^{-3} \mathrm{TECu} / \mathrm{nT}$. Magnetic pulsations ( $X$ component, KIR) and EISCAT electric field $E_{x}$ had coherency $\gamma \sim 0.8$. The cross-correlation between TEC variations from GPS9/KIRU and EISCAT field $E_{x}$ had a high coherency $\gamma(f) \sim 0.86$. The ratio between spectral amplitudes at this frequency was $\Delta N_{\mathrm{T}}(f) / E_{x}(f) \sim 4 \times 10^{-3} \mathrm{TECu} /(\mathrm{mV} / \mathrm{m})$.

An important parameter of ULF wave structure is its scale in the latitudinal (radial) and longitudinal (azimuthal) directions. The longitudinal propagation features are characterized by the azimuthal wave number $m$, which can be determined from a cross-correlation time shift $\Delta \tau$ between two detrended time series with $T$-periodic variations at sites separated in longitude by $\Delta \Lambda$, as follows $m=(\Delta \tau / T)\left(360^{\circ} / \Delta \Lambda\right)$. The cross-correlation function $R(\Delta \tau)$ for magnetic and TEC variations during time interval 1100-1130 UT has been estimated using the magnetic stations KIR-LOZ at geographic 
latitude $\sim 67.8^{\circ}$, longitudinally separated in geographic coordinates by $\Delta \Lambda \sim 15.4^{\circ}$, and the longitudinally separated pierce points along receiver/satellite paths TROM/ GPS9 and VARS/GPS28 at geographic latitude $\sim 69.7^{\circ}$ and separated in longitude by $\Delta \Lambda=27.2^{\circ}$ (Fig. 1). The

\section{Possible mechanisms of TEC modulation by MHD waves}

The temporal variations in the TEC evaluated along the line between a source $(S)$ and receiver $(R), N_{\mathrm{T}}(t)$, is given by the linearized path-integrated electron continuity equation

$$
\partial_{t} N_{\mathrm{T}}=\int_{S}^{R} \partial_{t} N(z) \mathrm{d} z=-\int_{S}^{R}\left[V_{z} \partial_{z} N_{0}+\mathbf{V}_{\perp} \nabla N_{0}+N_{0} \nabla \mathbf{V}\right] \mathrm{d} z+\langle Q\rangle-\langle L\rangle
$$

cross-correlation function of both magnetic and TEC variations has asymmetric form (Fig. 3), indicating westward propagation. The 10-s magnetic data reveal time delay $\Delta \tau=15 \mathrm{~s}$ (Fig. 3, upper panel). The correlation threshold at $95 \%$ confidence level, estimated by means a Monte Carlo test (Regi et al. 2015), at this lag is $\sim 0.2$. Thus, the cross-correlation coefficient $\sim 0.9$ is statistically significant. Though the $30-\mathrm{s}$ time resolution of TEC data is not sufficient to determine exactly a time shift, $R(\Delta \tau)$ reaches extreme values $R_{\max } \sim 0.8$ also around $\Delta \tau \sim 15 \mathrm{~s}$ (Fig. 3, bottom panel). For the wave frequency $f \sim 2.5 \mathrm{mHz}(T \sim 400 \mathrm{~s})$ this time shift corresponds to the azimuthal wave number (in geographic coordinates) $m \approx 0.9$ for magnetic data and $m \approx 0.5$ for TEC data. However, assuming that the error on the TEC delay time $\Delta(\Delta \tau)=15 \mathrm{~s}$ the estimated azimuthal wave number $m$ has relative error $\Delta m / m \sim 100 \%$. Thus, both magnetic and TEC data show a Pc5 wave propagation in the same westward direction, and no reliable conclusion about correspondence of the $m$ values from ionospheric TEC data and geomagnetic data can be made.

To find out which altitudes contributes most to the TEC variations, we have integrated ionospheric $N_{\mathrm{e}}(z)$ data from EISCAT over two different altitude range: the bottom ionosphere from 103 to $152 \mathrm{~km}$ and the F-layer from 152 to $415 \mathrm{~km}$. The height-time diagram of $N_{\mathrm{e}}(t)$ variations and altitude-integrated ionospheric densities $\left\langle N_{\mathrm{e}}\right\rangle$ (in TECu) are compared with actual vTEC variations for two time intervals: 1100-1200 UT for GPS9 (Fig. 4) and 1250-1350 UT for GPS7 (Fig. 5). Though short-lived Pc5 geomagnetic pulsation burst during 1220-1240 UT is also accompanied by TEC variations, the response at EISCAT is not very clear, so this time interval has not been included in a detailed analysis. Comparison of the EISCAT-derived quasi-TEC fluctuations $\left\langle N_{\mathrm{e}}\right\rangle$ with periodic vTEC variations shows that a closest match is observed for the bottom ionosphere, evidencing that the main contribution is provided by the lower ionosphere, up to $\sim 150 \mathrm{~km}$ (that is the E-layer and lower F-layer). However, the correspondence is not perfect, and maximal crosscorrelation coefficient between vTEC and $\left\langle N_{\mathrm{e}}\right\rangle$ is $R \sim 0.75$ for the first interval and $R \sim 0.72$ for the second interval. where $\langle Q\rangle$ and $\langle L\rangle$ are the height-integrated electron production and loss rates, respectively, $\mathbf{V}=\left\{V_{z}, \mathbf{V}_{\perp}\right\}$ is the plasma velocity from the ULF perturbation, and $N_{0}(z)$ is the background ionospheric density. Assuming $Q(z)$ and $L(z)$ are steady and equal, variations in TEC arise from the advection $\left(\propto \mathbf{V} \nabla N_{0}\right)$ and divergence $(\propto \nabla \mathbf{V})$ terms.

Variations in TEC along the signal path introduce time/ phase delays for high-frequency (HF) radio wave propagation through the ionosphere. The modulation of the ionospheric plasma density is due to the interaction of an incident MHD wave with the ionosphere-atmosphereground system. In this event no noticeable riometer variations with the same periodicity as geomagnetic pulsations were observed, so the mechanism of the periodic precipitation of energetic electrons is not considered, though the lack of soft $(<1 \mathrm{keV})$ electron precipitation cannot be guaranteed.

We use a standard model of the magnetosphere-ionosphere-atmosphere-ground system to describe the properties of MHD waves in a realistic ionosphere. We use the non-rectangular coordinate system $\left\{x_{1}, x_{2}, x_{3}\right\}$, where coordinate lines $x_{3}$ coincide with magnetic field lines, $x_{1}$ is measured along north-south direction, and $x_{2}$ is measured eastward. The atmosphere and the ground are assumed to be isotropic conductors with conductivities $\sigma_{\mathrm{a}}$ and $\sigma_{\mathrm{g}}$. The system parameters do not vary in the horizontal direction, i.e., along $x_{1}$ and $x_{2}$. The ionospheric plasma has anisotropic conductivity: Pedersen $\sigma_{\mathrm{p}}(z)$ and Hall $\sigma_{\mathrm{H}}(z)$. The magnetospheric plasma above the ionosphere is characterized by an Alfven velocity $\mathrm{V}_{\mathrm{A}}$ and wave number $k_{\mathrm{A}}=\omega / V_{\mathrm{A}}$.

ULF electromagnetic field inside the ionosphere is composed from Alfven and fast magnetosonic (compressional) waves, consisting of incident, reflected, and mutually converted modes. The coupled MHD equations describing these modes in an anisotropic collisional plasma can be found in Waters et al. (2007) and Fedorov et al. (2016). The vertical profile of local of the ionospheric parameters (conductivity tensor, Alfven velocity, electron mobility tensor, etc.) is to construct from an adequate ionospheric model. Further, it will be assumed that an incident ULF wave is the Alfven mode. 


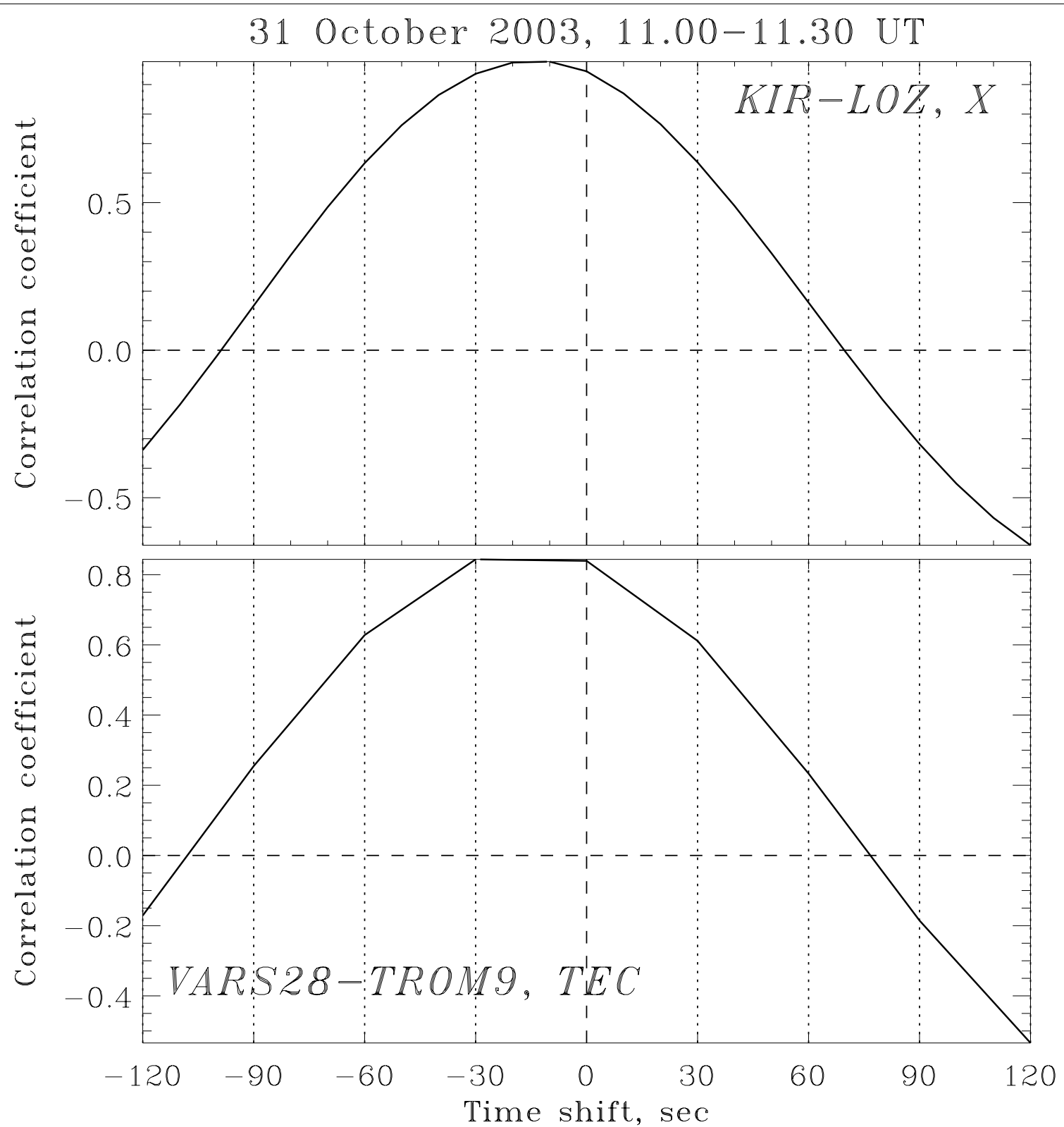

Fig. 3 The cross-correlation function $R(\Delta T)$ of magnetic variations (upper panel) during time interval 1100-1130 UT at stations KIR-LOZ ( $\left.\Phi \sim 67.8^{\circ}\right)$, longitudinally separated by $\triangle \Lambda \sim 15.4^{\circ}$ in geographic coordinates, and of TEC variations (bottom panel) determined from the longitudinally separated by $\Lambda=27.2^{\circ}$ in geographic coordinates pierce points $\left(\Phi \sim 68.7^{\circ}-69.8^{\circ}\right)$ along receiver/satellite paths TROM/GPS9 and VARS/GPS28

The TEC and magnetometers observations at longitudinally widely separated sites have shown a large azimuthal scale of both ionospheric and magnetic Pc5 pulsations, corresponding to $m \leq 1$. Therefore, it may be supposed that $k_{2} \sim m \rightarrow 0$. The scale in radial (latitudinal) direction is characterized by the wave vector $k \cong k_{1}$. The incident Alfven mode elongated in the azimuthal direction has an azimuthal magnetic component $b_{2}$ only, whereas the wave electric field component $E_{1}$ lies in the meridional plane and is transverse to $\mathbf{B}_{0}$. The fast magnetosonic (FMS) mode has non-vanishing azimuthal electric component $E_{2}$, magnetic radial $b_{1}$, and compressional $b_{3} \equiv b_{\|}$components.

The components of electron velocity induced by the wave electric field $\mathbf{E}$ are determined by local plasma mobility tensor $\hat{\mu}^{(\mathrm{e})}$ 


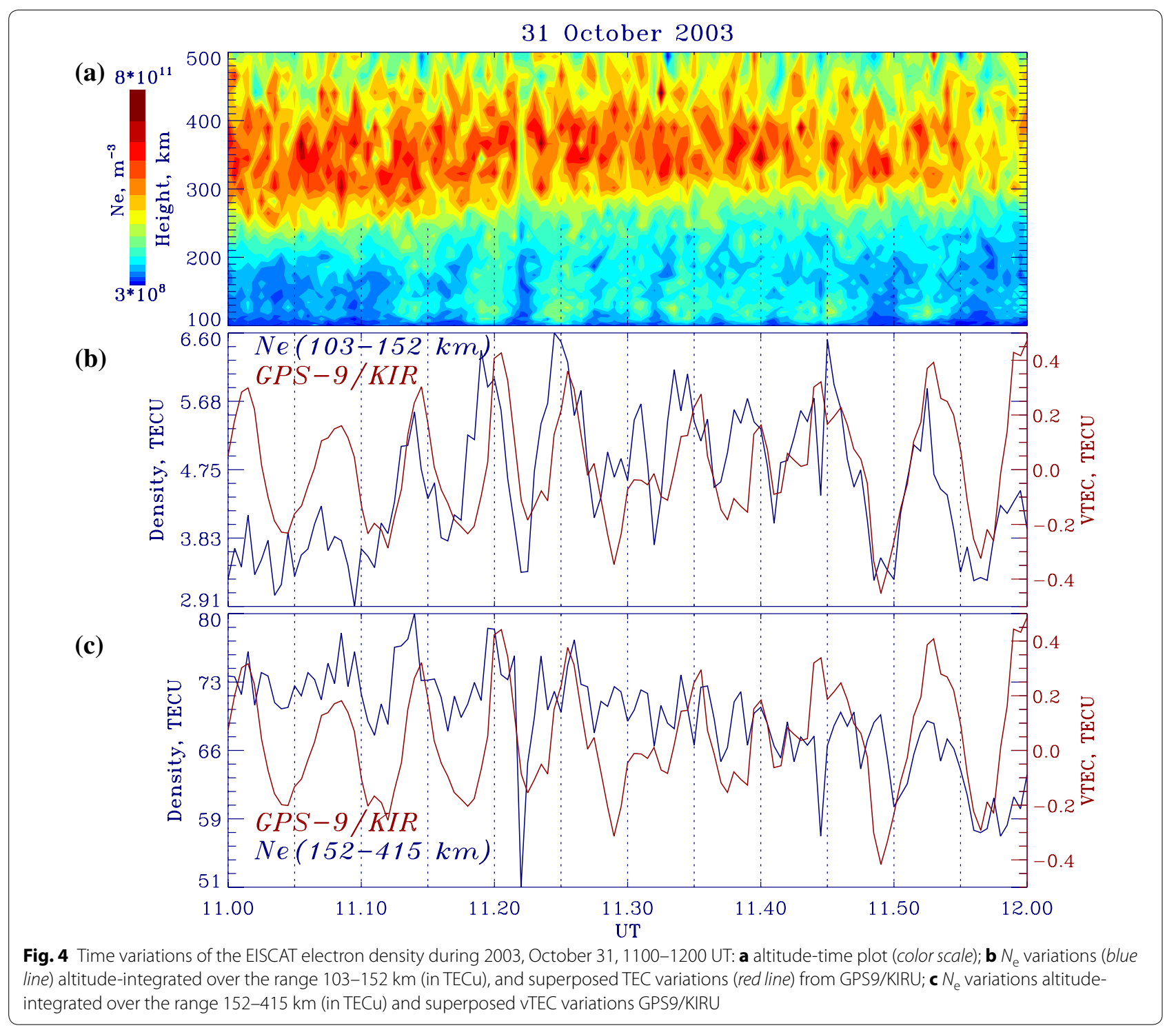

$$
\begin{aligned}
& V_{1}^{(\mathrm{e})}=\mu_{1}^{(\mathrm{e})} E_{1}+\mu_{2}^{(\mathrm{e})} E_{2} \\
& V_{2}^{(\mathrm{e})}=-\mu_{2}^{(\mathrm{e})} E_{1}+\mu_{1}^{(\mathrm{e})} E_{2} \\
& V_{3}^{(\mathrm{e})}=\mu_{3}^{(\mathrm{e})} E_{3}
\end{aligned}
$$

The corresponding electron density perturbation $N^{(\mathrm{e})}=N_{1}^{(\mathrm{e})}+N_{2}^{(\mathrm{e})}+N_{3}^{(\mathrm{e})}$ is produced by three currents (Pedersen, Hall, and parallel) as follows

$\frac{N_{1}^{(\mathrm{e})}}{N_{0}}=\frac{k}{\omega} \mu_{1}^{(\mathrm{e})} E_{1}, \quad \frac{N_{2}^{(\mathrm{e})}}{N_{0}}=\frac{k}{\omega} \mu_{2}^{(\mathrm{e})} E_{2}, \quad \frac{N_{3}^{(\mathrm{e})}}{N_{0}}=\frac{1}{i \omega} \partial_{3} \mu_{3}^{(\mathrm{e})} E_{3}$

The ion density perturbation $N^{(\mathrm{i})}=N_{1}^{(\mathrm{i})}+N_{2}^{(\mathrm{i})}+N_{3}^{(\mathrm{i})}$ keeps the plasma electroneutrality.
The magnetic field and plasma compression can be produced by an incident fast mode wave $\Delta N_{\mathrm{T}} / N_{\mathrm{T}} \simeq b_{\|} / B_{0}$. Such effect of the ionospheric plasma periodic compression by Pc 5 pulsations, associated with the fast mode, was indeed observed with GPS observations at low latitudes, where Pc5 wave frequency is much lower than the Alfven field line eigenfrequency (Vorontsova et al. 2016).

However, even an incident Alfven wave can produce a secondary fast compressional mode upon the interaction with the anisotropic ionosphere. This evanescent fast mode wave is excited in the ionosphere by incident Alfven wave owing to the ionospheric Hall conductance. Thus, TEC modulation may be related to the compression of plasma caused by this secondary 


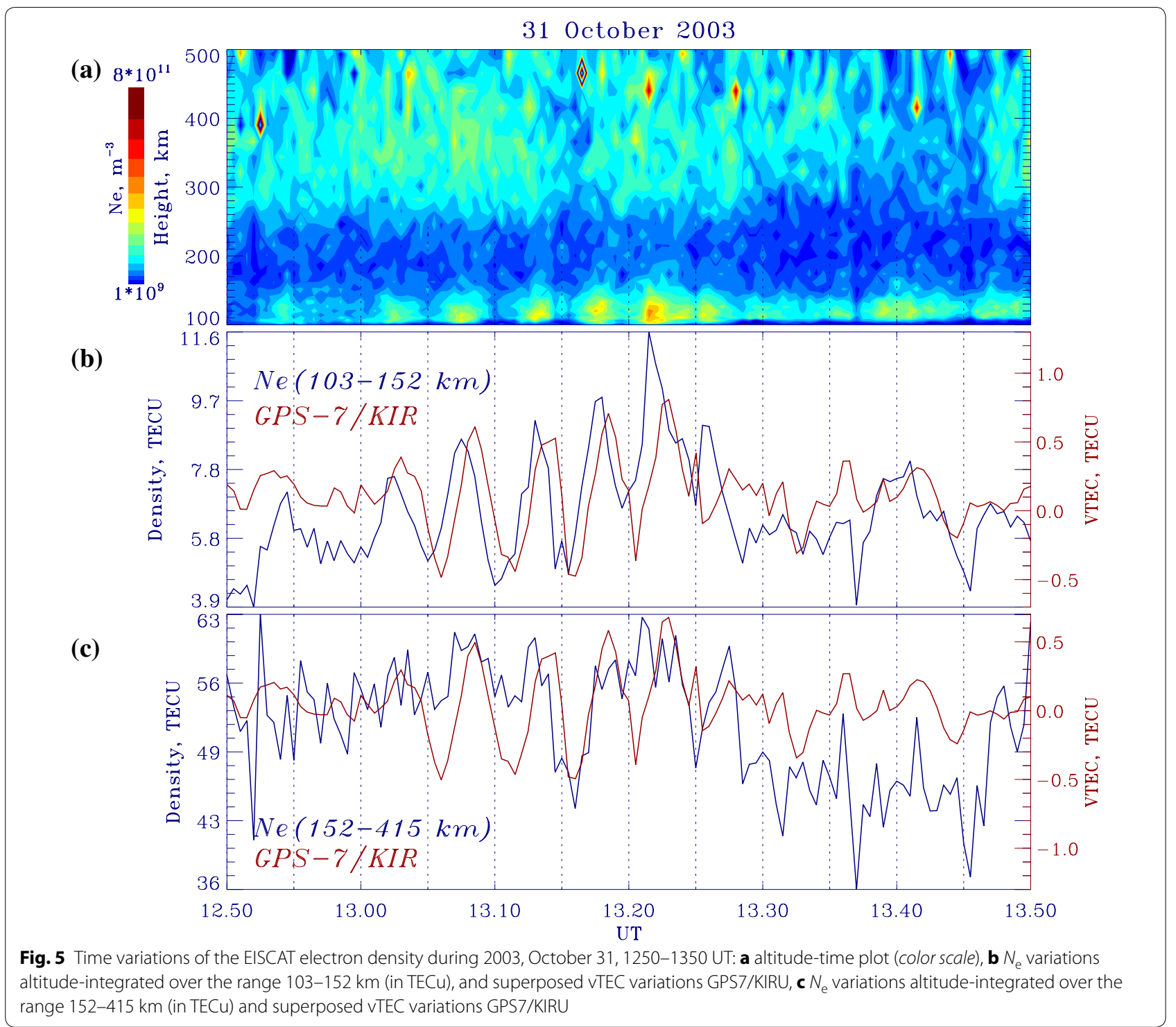

fast compressional mode [the term $N_{2}$ in (3)]. Indeed, if we substitute $E_{2}=(\omega / k) b_{3}$ in $N_{2}=\frac{k}{\omega} \frac{N_{0}}{B_{0}} E_{2}$, we obtain $\frac{N_{2}}{N_{0}}=\frac{b_{3}}{B_{0}}$, where $b_{3}$ is the compressional magnetic component. Pilipenko and Fedorov (1995) estimated the modulation of plasma owing to a partial conversion of an incident Alfven wave into an evanescent fast mode wave and showed that it might be significant for small-scale incident waves. Such small-scale waves are to be screened by the ionosphere from ground magnetometers.

Other possible mechanism of TEC modulation by an incident Alfven wave (Waters and Cox 2009) may comprise a periodic drift (advection) across a lateral gradient of the ionospheric plasma [second righthand term in (1)]. This mechanism can be expected to be important only for more localized and steep ionospheric inhomogeneities. We have no information about lateral gradients of $N_{\mathrm{e}}$ during this event. However, the largest effect produced by this mechanism is expected to be in the F-layer, where plasma concentration is highest.

The finite east-west $E_{2}$ component of an incident Alfven wave causes a vertical plasma drift $V_{z}=E_{2} \cos I / B_{0}$, where $I$ is the geomagnetic field inclination. This vertical shift causes a plasma modification due to the changes of ionization-recombination balance owing to a strong dependence of the ionization $Q(z)$ and recombination $L(z)$ rates on altitude (Poole and Sutcliffe 1987). Periodic vertical shift of ionospheric plasma, which is accompanied by a reconfiguration of the 
ionization-recombination balance, can provide a noticeable contribution to the TEC modulation by Pc5 electric field, but around the maximum of ionization (F-layer). Though, $E_{2}$ component of the large-scale Alfven wave $\left(k_{2} \rightarrow 0\right)$ is to be small.

The periodic heating of ionospheric ions can occur during times when plasma is dragged through neutrals by the Pc5 wave electric field (Lathuillere et al. 1986). This additional plasma heating may shift the ionizationrecombination balance due to the dependence of the recombination coefficient $\beta(T)$ on temperature and cause plasma density variations (Pilipenko et al. 2014a). The periodic ion heating in the bottom ionosphere during October 31, 2003, event by Pc5 wave electric field indeed can be seen in the EISCAT data (Pilipenko et al. 2014b).

In a realistic ionosphere all the above mechanisms may operate simultaneously, so it is hard to distinguish their contribution into the TEC variations and to compare their efficiency, because many specific parameters are not well known for an event under study. Combined EISCAT and TEC observations have indicated that the plasma modulation by Pc 5 wave is most significant in the lower ionosphere. This fact contradicts the predictions of TEC modulation theories, based on the F-layer vertical shift and lateral gradient. At the same time, this observational fact favors the mechanism of field-aligned plasma transfer induced by Alfven wave. Further we present a simple theoretical model to examine this mechanism in a greater detail.

\section{Field-aligned plasma transport}

Though all of the above-mentioned mechanisms may somewhat contribute to the periodic TEC variations, here we concentrate on another possible mechanism, related to field-aligned plasma transport (Cran-McGreehin et al. 2007). To give an insight into its basic physics, we first provide a simple analytical estimate in a model with vertical geomagnetic field $\mathbf{B}_{0}=B_{0} \mathbf{e}_{3}$. The fieldaligned current $j_{3} \equiv j_{Z}$ transported by an Alfven wave, incident onto the ionosphere from the magnetosphere, provides an additional periodic plasma flow in/out the ionosphere. As a result, the plasma density in the bottom ionosphere periodically increases/decreases. An upper estimate of this effect can be obtained from the heightintegrated balance equation

$$
\partial_{t} N_{\mathrm{T}}=j_{3} / e
$$

where $e=1.6 \times 10^{-19} \mathrm{C}$ is the electron charge. The electron transverse diffusion across $\mathbf{B}_{0}$ during a wave period is assumed to be small. From Eq. (4) it follows that an oscillating current $j_{3}=j_{3}^{(0)} \exp (-i \omega t)$ causes periodic fluctuations of TEC with amplitude $\Delta N_{\mathrm{T}}=i j_{3}^{(0)} / e \omega$. The current $j_{3}$ transported by an Alfven wave is related to the wave magnetic field $b_{2}$ in the ionosphere as follows $j_{3}=-i k b_{2} / \mu_{0}$. Combination of these two relationship yields

$$
\Delta N_{\mathrm{T}}=\frac{k b_{2}}{e \mu_{0} \omega}=\frac{T}{e \mu_{0} \lambda} b_{2}
$$

where $T$ is the wave period and $\lambda$ is the wave latitudinal scale corresponding to the characteristic wave number $k \sim 2 \pi / \lambda$. The relationship (5) gives an upper-limit order of magnitude estimate of the ionospheric effect. Let us suppose that a typical peak-to-peak $H$-component amplitude of global 400-s Pc5 pulsations on the ground is $b_{1}^{(\mathrm{g})} \simeq 400 \mathrm{nT}$. For simplicity, a radio path between a satellite and ground receiving station is assumed to be vertical. Because the transverse scale of global Pc5 waves under study, $\sim 10^{3} \mathrm{~km}$ (Kleimenova and Kozyreva 2005), is much bigger than the height of the ionospheric conductive layer, $\sim 100 \mathrm{~km}$, the magnetic field in the ionosphere is related to the response on the ground as $b_{1}^{(\mathrm{g})} / b_{2} \simeq\left(\Sigma_{\mathrm{H}} / \Sigma_{\mathrm{P}}\right) \sin I$ (Hughes and Southwood 1976). For typical at high latitudes $\Sigma_{\mathrm{H}} / \Sigma_{\mathrm{P}} \sim 1.6$, and $\sin$ $I \sim 0.9$, the magnetic field disturbance in the ionosphere is $b_{2} \sim 290 \mathrm{nT}$. According to (5) an Alfven wave with the given amplitude should cause fluctuations in the bottom ionosphere with $\Delta N_{\mathrm{T}} \sim 0.2 \mathrm{TECu}$. This order-ofmagnitude estimate indicates that the effect of periodic pumping/depleting into the lower ionosphere of the field-aligned electron flux, transported by an Alfven wave, in principle can be responsible for the TEC modulation by global Pc5 pulsations. In reality, the TEC modulation rate is determined by a spatial integration along a radio path of local plasma response to an inhomogeneous wave E-field and thus should be strongly dependent on the satellite elevation angle and wave transverse scale. These factors can be taken into account with the help of a numerical model only.

\section{Numerical model of Alfven wave interaction with the realistic ionosphere}

The above simplified estimate has been validated with numerical modeling. We consider the incidence of Alfven wave with a large azimuthal scale $\left(k_{2}=0\right)$ onto the ionosphere. For such wave structure the E-W electric field component $E_{2} \rightarrow 0$, so the mechanism of vertical drift is inoperative. The heating of the ionosphere by wave has been neglected as well. Thus, this simplified case enables us to isolate and examine the mechanism of the fieldaligned plasma transport.

The ionospheric medium parameters have been derived from the IRI-2012 model. The modeling procedure comprises the following steps: (1) For a given geophysical conditions the IRI-derived altitude profile $(80-2000 \mathrm{~km})$ of ionospheric parameters is constructed; (2) the wave 
electric $\mathbf{E}(z)$ and magnetic $\mathbf{b}(z)$ field vertical structure is calculated using the numerical solution of a set of coupled MHD wave equations in the ionosphere, whereas incidence of Alfven wave with horizontal wave vector $k$ has been assumed; (3) using the mobility tensor the plasma vertical and horizontal fluxes are determined via (2) throughout the ionosphere; (4) using the continuity equation the local disturbance of plasma density $N_{\mathrm{e}}(z)$ is determined; and (5) the vertical structure of local plasma density disturbance is height-integrated to provide a disturbance of TEC, $N_{\mathrm{T}}$. The magnetic field geometry (inclination of $\mathbf{B}_{0}$ is $I=61.9^{\circ}$ ) and IRI parameters correspond to the observational period 2003.10.31, 11.5 UT near TRO. The IRI model yields the height-integrated Pedersen and Hall conductance as follows $\Sigma_{\mathrm{P}}=2.2 \mathrm{~S}$ and $\Sigma_{\mathrm{H}}=3.6 \mathrm{~S}$.

When MHD waves interact with the ionosphere, their wave properties are modified. The calculated vertical structure of the wave electric field for various frequencies of incident Alfven wave with transverse wave vector $k=10^{-3} \mathrm{~km}^{-1}$ is shown in Fig. 6. For a chosen azimuthally large-scale structure, the $\mathrm{N}-\mathrm{S}$ electric field component is dominating, $E_{1} \gg E_{2}$. Along $z$ the $E_{1}(z)$ component is nearly constant thanks to the field line equipotentiality. A weaker $E_{2}$ component of a secondary fast mode emerges thanks to the mode coupling in the anisotropically conductive ionosphere. The fast mode is evanescent and decays with altitude.

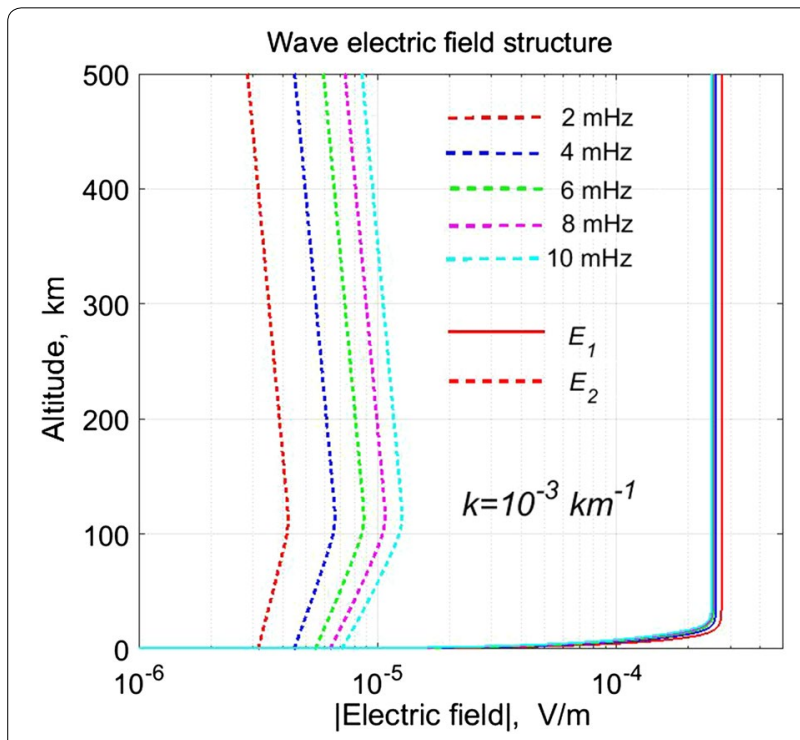

Fig. 6 Wave electric field vertical structure, produced by incident Alfven wave with $k=10^{-3} \mathrm{~km}^{-1}$ : Solid lines correspond to $E_{1}$ component, and dashed lines correspond to $E_{2}$ component. Different colors denote different frequencies (shown in the inset). All wave fields in the ionosphere have been normalized to the magnitude of the total horizontal magnetic field on the ground $b_{1}=1 \mathrm{nT}$
Local disturbances of electron density $N_{\mathrm{e}}(z)$, induced by the wave $E$-field, are shown in Fig. 7. The fieldaligned electron fluxes result in enhancements of $N_{\mathrm{e}}$ in the E-layer and bottom of F-layer. The vertical profile of disturbed plasma density $N_{\mathrm{e}}(z)$ has local maxima at altitudes $\sim 300, \sim 200 \mathrm{~km}$, and order of magnitude larger at $\sim 120 \mathrm{~km}$. Calculations show that the contribution to the electron plasma density disturbance by the Pedersen current and plasma compression are small as compared with the contribution from the field-aligned electron flux. Thus, the periodic field-aligned electron flux produces local variations of electron density in the E-layer and bottom of the F-layer.

The resultant height-integrated effect is presented in Fig. 8. This figure shows the calculated dependence of the TEC amplitude fractional modulation, $\Delta N_{\mathrm{T}} / N_{\mathrm{T}}$, on the transverse wave number $k$ induced by an incident Alfven wave with total horizontal magnetic component on the ground $b_{1}=1 \mathrm{nT}$ (peak-to-peak amplitude $2 \mathrm{nT}$ ) for various frequencies. In the range up to $k=10^{-2} \mathrm{~km}$ the TEC modulation rate increases with $k$ owing to the increase of the wave-transported field-aligned current (Fig. 8a). The modulation rate is higher for lower frequencies. For $k=0.006 \mathrm{~km}$ (wave scale $\sim 2 \pi / k \sim 10^{3} \mathrm{~km}$ ) the predicted TEC modulation depth is $\sim 0.025 \%$. Therefore, for peakto-peak $400 \mathrm{nT}$ Pc5 pulsations, recorded during October 31, 2003, event, the TEC fluctuations are expected to be $\sim 5 \%$. This value is about the observed peak magnitudes during the analyzed event: $N_{\mathrm{T}} \sim 40 \mathrm{TECu}, \Delta N_{\mathrm{T}} \sim 1$ $\mathrm{TECu}$, hence $\Delta N_{\mathrm{T}} / N_{\mathrm{T}} \sim 2.5 \%$. The theoretical value is

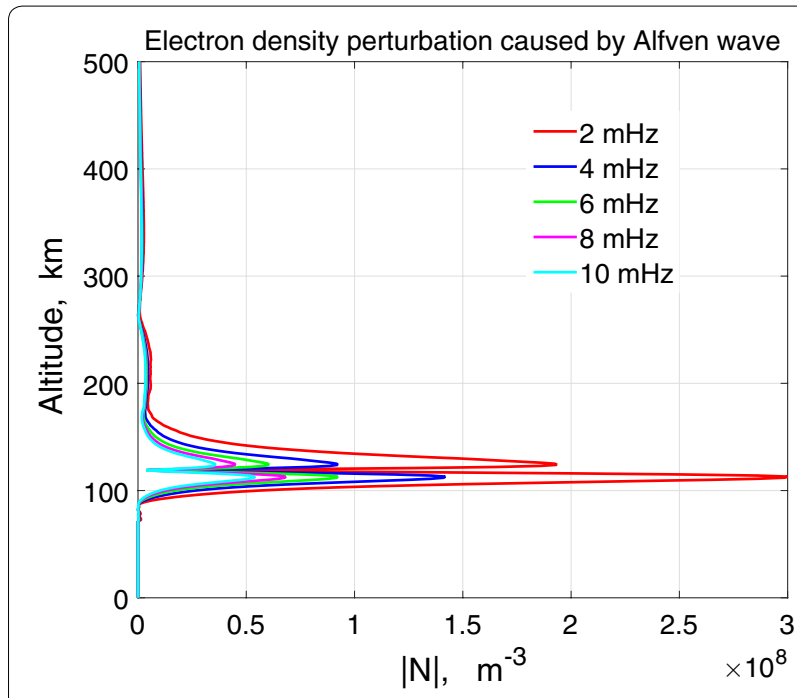

Fig. 7 Wave-induced plasma density perturbation, produced by incident Alfven wave with $k=10^{-3} \mathrm{~km}^{-1}$. Different colors denote different frequencies (shown in the legend) 


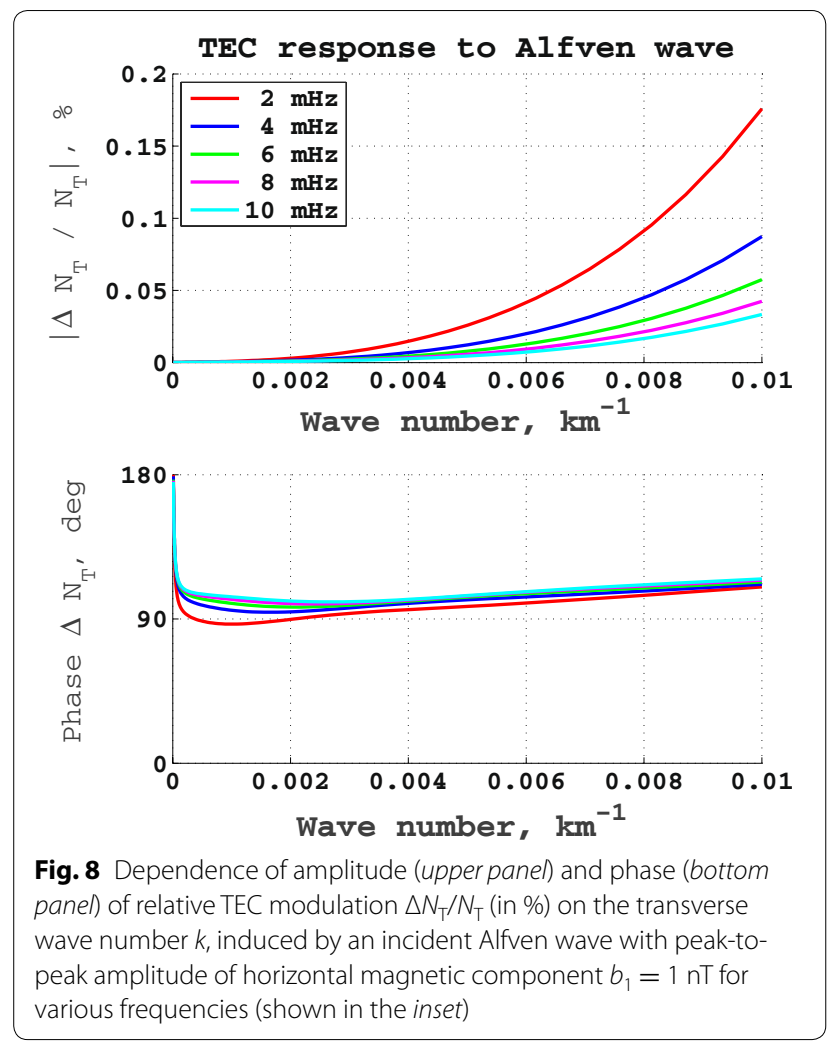

probably somewhat overestimated because only the vertical radiopath satellite-receiver is considered.

Our idealized model predicts a phase shift between the TEC variations and ground magnetic pulsations $(H$ component) about $\Delta \phi \sim 100^{\circ}$ (Fig. 8b). However, a crossspectral estimate of $\Delta \phi(f)$ is not very reliable because the phase delay between TEC and $B$ time series fluctuates considerably during the analyzed events. Therefore, the relative phase information cannot tell anything definitive to be compared with the model prediction.

\section{Discussion}

Our current knowledge of the ULF wave physics has been acquired mainly with the help of ground-based or satellite-borne magnetometers. However, their capabilities are limited, because even low-orbiting satellites cannot detect in situ long-period ULF waves in the ionosphere-the region where energy flows from the magnetosphere into the upper atmosphere. Moreover, the ionosphere screens transversely small-scale structures $(<100 \mathrm{~km})$ from ground magnetometers. Ionospheric radars have emerged as a valuable source of additional information for ULF wave studies. GPS/TEC observations are expected to provide similarly new information about MHD wave interaction with the ionosphere.
Long-period pulsations are the most powerful wave process in the near-Earth environment. The radar observations showed that Pc5 waves can noticeably modulate the ionospheric plasma: the electric field $\mathbf{E}$, plasma convection velocity $\mathrm{V}$, E-layer electron density $N_{\mathrm{e}}$ and the ionosphere conductance $\Sigma$, and electron $T_{\mathrm{e}}$ and ion $T_{\mathrm{i}}$ temperatures in both F- and E-layers (see references in Pilipenko et al. 2014b). Recent observations by Pilipenko et al. (2014a) and Watson et al. (2015) have demonstrated that Pc5 waves are capable to modulate TEC as well.

One may expect that all the Pc5 wave-induced fractional variations of plasma and magnetic field should be of the same magnitude, like in any linear wave. However, GPS observations have revealed that the depth of periodic TEC modulation is sometimes even somewhat larger (e.g., in the event of October 31, 2003, $\Delta N_{\mathrm{T}} / N_{\mathrm{T}} \sim 2.5 \%$ ) than the geomagnetic field modulation $\left(\triangle B / B_{0} \sim 1 \%\right)$. In principle, ULF modulation of energetic electron precipitation, inducing an additional periodic ionization of the lower ionosphere, can cause periodic TEC variations with much higher depth than geomagnetic field variations (Watson et al. 2015). However, during the event under consideration no periodic electron precipitation occurred as evidenced by simultaneous riometer observations. The mechanism of the field-aligned plasma transport by Alfven waves, described in "Numerical model of Alfven wave interaction with the realistic ionosphere" section, can theoretically produce relative amplitudes of TEC variations larger than that of geomagnetic pulsations. However, we have analyzed GPS/TEC data during an event with very intense Pc5 waves. Whether TEC modulation by less intense ULF waves would be revealed by standard GPS technique is an open question.

Consideration of possible mechanisms of TEC modulation by a magnetospheric Alfven wave ("Possible mechanisms of TEC modulation by MHD waves" section) has shown that in principle the plasma heating, vertical drift, steep gradient, and field-aligned transport can provide a noticeable input into the TEC variations. In some cases, a periodically modulated precipitation of magnetospheric electrons can be effective, too. A feature of the field-aligned electron transport mechanism is that it contributes mainly into the plasma density of the bottom ionospheric layers. This feature is basically in accordance with the combined GPS/EISCAT/magnetometer observations. However, as the correlation between $\left\langle N_{\mathrm{e}}\right\rangle$ and vTEC is $R \sim 0.75$, the field-aligned transport mechanism is responsible for $\sim 56 \%$ of TEC variations. Thus, though the proposed hypothesis on field-aligned plasma transport is basically consistent with the analyzed event, the correspondence between magnetometer, EISCAT, and GPS/TEC oscillations is not always perfect. For 
example, short-lived Pc5 geomagnetic pulsations during 1220-1240 UT is accompanied by TEC variations, but the response at EISCAT is not clear. Probably, some of the mentioned above mechanisms also contribute to TEC periodic modulation by ULF wave, though a limited available information does not enable us to single them out.

The phase relation between magnetic and TEC variations is not very stable and does not allow to use it for the model validation and discrimination of possible mechanisms. Moreover, the phase information is rather subtle, and its consideration must be done with a great caution. The developed model is still too simplified: Pc5 transverse spatial structure is modeled as plane wave, and TEC is calculated along a vertical path. Therefore, the phase information can be used as a tool for the discrimination of possible mechanisms only on the basis of a more advanced model. More conclusive judgments can be stated only after detailed studies with the use of other ionospheric instruments that will provide additional information about ionospheric plasma parameters and incident particle fluxes.

\section{Conclusion}

Long-period geomagnetic Pc5 pulsations being the most powerful wave process in the terrestrial environment can significantly modulate the local densities of the magnetospheric and ionospheric plasma. Even radiopathintegrated TEC has turned out to be sensitive enough to respond to intense Pc5 waves. So far, the effect of TEC modulation by ULF waves is a challenge for the MHD wave theory, because responsible mechanisms of such modulation have not been firmly established yet. Analysis of the altitude profile of the electron density fluctuations derived from EISCAT data during the global Pc5 wave event has shown that main contribution into the periodic TEC variations is provided by lower ionosphere, up to $\sim 150 \mathrm{~km}$, that is the E-layer and lower F-layer. This observational fact favors the field-aligned plasma transfer induced by Alfven wave as a dominant modulation mechanism. The analytical estimate and numerical modeling have shown a high efficiency of this mechanism. However, sometimes the correspondence between magnetometer, EISCAT, and GPS/TEC oscillations is not perfect, which indicates that some other modulation mechanisms could come into play.

\section{Authors' contributions \\ BV made GPS and magnetometer data analysis, VP made analytical estimates and drafted the manuscript, DM participated in the GPS and riometer data analysis, EF performed numerical modeling, AK carried out the EISCAT data analysis. All authors read and approved the final manuscript.}

\section{Author details}

${ }^{1}$ Polar Geophysical Institute, Apatity, Russia. ${ }^{2}$ Space Research Institute, Moscow, Russia. ${ }^{3}$ Augsburg College, Minneapolis, MN, USA. ${ }^{4}$ Institute of Physics of the Earth, Moscow, Russia. ${ }^{5}$ Sodankyla Geophysical Observatory, University of Oulu, Oulu, Finland.

\section{Acknowledgements}

This study was funded by the RF President Grant MK-4210.2015.5 (BV), RFBR Grants 14-05-00588 (VP), 15-05-01814 (EF), and NSF Grant ATM-0827903 to Augsburg College (DM). Dual-frequency 30-s rate GPS daily data files in RINEX format are freely available from the IGS (ftp://cddis.gsfc.nasa.gov). We are indebted to the staff of EISCAT for operating the facility and supplying the data. We thank the institutes who maintain the IMAGE magnetometer array (www.ava.fmi.fi/image). The riometer data originated from the IRIS, operated by the Lancaster University in collaboration with the Sodankyla Geophysical Observatory. We appreciate detailed comments of both reviewers.

Received: 19 December 2015 Accepted: 25 May 2016

Published online: 07 June 2016

\section{References}

Afraimovich EL, Astafyeva El, Demyanov W et al (2013) A review of GPS/GLONASS studies of the ionospheric response to natural and anthropogenic processes and phenomena. J Space Weather Space Clim 3:A27

Cran-McGreehin AP, Wright AN, Hood AW (2007) Ionospheric depletion in downward currents. J Geophys Res 112:A10309

Davies K, Hartman GK (1976) Short-period fluctuations in total columnar electron content. J Geophys Res 81:3431-3434

Fedorov E, Mazur N, Pilipenko V, Engebretson M (2016) Interaction of magnetospheric Alfven waves with the ionosphere in the Pc1 frequency band. J Geophys Res 121:321-337

Hughes WJ, Southwood DJ (1976) The screening of micropulsation signals by the atmosphere and ionosphere. J Geophys Res 81:3234-3240

Kleimenova NG, Kozyreva OV (2005) Spatial-temporal dynamics of Pi3 and Pc5 geomagnetic pulsations during the extreme magnetic storms in October 2003. Geomagn Aeron (Engl Transl) 45:71-79

Komjathy A, Galvan DA, Stephens P et al (2012) Detecting ionospheric TEC perturbations caused by natural hazards using a global network of GPS receivers: the Tohoku case study. Earth Planets Space 64:1287-1294

Lathuillere C, Glangeaud F, Zhao ZY (1986) lonospheric ion heating by ULF PC5 magnetic pulsations. J Geophys Res 91:1619-1626

Lester M, Davies JA, Yeoman TK (2000) The ionospheric response during an interval of PC5 ULF wave activity. Ann Geophys 18:257-261

Marin J, Pilipenko V, Kozyreva O, Stepanova M, Engebretson M, Vega P, Zesta E (2014) Global Pc5 pulsations during strong magnetic storms: excitation mechanisms and equatorward expansion. Ann Geophys 32:319-331

Menk FW, Waters CL, Dunlop SI (2007) ULF Doppler oscillations in the low latitude ionosphere. Geophys Res Lett 34:L10104. doi:10.1029/200 7GL029300

Okuzawa T, Davies K (1981) Pulsations in the total columnar electron content. J Geophys Res 86:1355-1363

Pilipenko V, Fedorov E (1995) Modulation of total electron content in the ionosphere by geomagnetic pulsations. Geomagn Aeron (Engl Transl) 34:516-519

Pilipenko V, Belakhovsky V, Kozlovsky A, Fedorov E, Kauristie K (2012) Determination of the wave mode contribution into the ULF pulsations from combined radar and magnetometer data: method of apparent impedance. J Atmos Sol-Terr Phys 77:85-95

Pilipenko VA, Fedorov EN, Teramoto M, Yumoto K (2013) The mechanism of mid-latitude Pi2 waves in the upper ionosphere as revealed by combined Doppler and magnetometer observations. Ann Geophys 31:689-695

Pilipenko V, Belakhovsky V, Murr D, Fedorov E, Engebretson M (2014a) Modulation of total electron content by ULF PC5 waves. J Geophys Res 119:4358-4369

Pilipenko V, Belakhovsky V, Kozlovsky A, Fedorov E, Kauristie K (2014b) ULF wave modulation of the ionospheric parameters: radar and magnetometer observations. J Atmos Sol-Terr Phys 108:68-76

Ponomarenko PV, Waters CL, Sciffer MD, Fraser BJ (2001) Spatial structure of ULF waves: comparison of magnetometer and Super DARN data. J Geophys Res 106:10509-10517 
Poole AWV, Sutcliffe PR (1987) Mechanisms for observed total electron content pulsations at mid latitudes. J Atmos Terr Phys 49:231-236

Potapov A, Guglielmi A, Tsegmed B, Kultima J (2006) Global Pc5 event during 29-31 October 2003 magnetic storm. Adv Space Res 38:1582-1586

Regi M, De Lauretis M, Francia P (2015) Pc5 geomagnetic fluctuations in response to solar wind excitation and their relationship with relativistic electron fluxes in the outer radiation belt. Earth Planets Space 67:9. doi:10.1186/s40623-015-0180-8

Teramoto M, Nishitani N, Pilipenko V et al (2014) Pi2 pulsation simultaneously observed in the $\mathrm{E}$ and $\mathrm{F}$ region ionosphere with the SuperDARN Hokkaido radar. J Geophys Res 119:3444-3462

Vorontsova E, Pilipenko V, Fedorov E, Sinha AK, Vichare G (2016) Modulation of total electron content by global Pc5 waves at low latitudes. Adv Space Res 57:309-319
Waters CL, Cox SP (2009) ULF wave effects on high frequency signal propagation through the ionosphere. Ann Geophys 27:2779-2788

Waters CL, Yeoman TK, Sciffer MD, Ponomarenko P, Wright DM (2007) Modulation of radio frequency signals by ULF waves. Ann Geophys 25:1113-1124

Watson C, Jayachandran PT, Singer HJ, Redmon RJ, Danskin D (2015) Largeamplitude GPS TEC variations associated with PC5-6 magnetic field variations observed on the ground and at geosynchronous orbit. J Geophys Res. doi:10.1002/2015JA021517

\section{Submit your manuscript to a SpringerOpen ${ }^{\odot}$ journal and benefit from:}

- Convenient online submission

- Rigorous peer review

- Immediate publication on acceptance

- Open access: articles freely available online

- High visibility within the field

- Retaining the copyright to your article 